\title{
GISE REPORT
}

\section{Clear cell predominant epithelial-myoepithelial carcinoma of the hard palate - role of immunohistochemistry}

\author{
Radha R. Pai · Kausalya Sahu · Anand U. Kini
}

\begin{abstract}
Epithelial-myoepithelial carcinoma (EMC) arising from the minor salivary gland of the hard palate is very rare. A 34-year-old lady presented with a nodular swelling in the hard palate. Histopathologically, a diagnosis of clear cell predominant EMC was suggested. Immunohistochemistry demonstrated the biphasic nature of the tumor and confirmed the diagnosis of EMC.
\end{abstract}

Keywords Hard palate · Palatal neoplasms · Epithelial-myoepithelial carcinoma

\section{Introduction}

Epithelial-myoepithelial carcinoma (EMC) is a rare biphasic, low-grade malignant tumour of the salivary gland. It constitutes $1-2 \%$ of salivary gland neoplasms [1]. The neoplasm demonstrates a slight predilection for females, with the average age being in the 6th to 7th decades of life [2]. Most tumours arise in the major salivary gland, especially parotid, but cases have been described in the submandibular gland, minor salivary glands of the oral cavity, nasal sinuses, bronchus, trachea, nasal cavity, external auditory canal,

\footnotetext{
R. R. $\mathrm{Pai}^{1} \cdot$ K. Sahu ${ }^{1} \cdot$ A. U. Kini ${ }^{2}$

${ }^{1}$ Departments of Pathology

${ }^{2}$ Surgery

Kasturba Medical College

Mangalore, India

R. R. Pai $(\bowtie)$

Additional Professor, Department of Pathology,

Kasturba Medical College, P. O. Box 53,

Mangalore - 575001

Karnataka, India

e-mail: pairadharam@yahoo.co.in

Tel: 0824-2423452

Fax: +91 / 824 / 428183
}

and, lacrimal gland. Most recently it is described in the liver [1]. It has been postulated that the tumour is of intercalated ductal origin. Intercalated duct hyperplasia may be a precursor lesion to EMC and other salivary gland carcinomas [1]. When the biphasic pattern is less apparent immunohistochemistry plays a role to demonstrate the coexistence of glandular and myoepithelial components.

\section{Case report}

A 36-year-old lady presented with swelling in the hard palate of 1 month duration. There was no history of pain. On examination, a nodular swelling was seen in the hard palate in the left maxillary molar region with surface ulceration. The nodule was extending onto the gingiva with loss of adjacent tooth. There was no evidence of cervical lymphadenopathy. All other systems were within normal limits. Clinically, a differential diagnosis of minor salivary gland neoplasm and epulis was considered.

On incisional biopsy solid nests of mildly pleomorphic polygonal cells were seen and a diagnosis of carcinoma, probably mucoepidermoid was suggested. Wide excision with partial maxillectomy was performed.

Grossly, the partial maxillectomy specimen measured $4 \times 3$ $\times 2 \mathrm{cms}$ with a nodular growth $2 \mathrm{~cm}$ in diameter extending from the hard palate to the gingiva in the region of first molar. The cut surface showed a circumscribed homogenous, solid, gray-white multinodular tumor extending upto the periosteum. The overlying mucosa was ulcerated.

Histopathologically, sections showed ulcerated squamous epithelium with an underlying multinodular tumor with apparent circumscription. The tumor was made up of round to oval cells with moderate amount of pale pink to clear cytoplasm and round to oval eccentrically placed mildly pleomorphic vesicular nuclei with small prominent nucleoli. An occasional mitotic figure was seen. The tumor cells were arranged in an organoid and trabecular fashion 
separated by hyalinized connective tissue stroma. Focally solid groups of polygonal clear cells some with central small mucin containing luminal spaces lined by flattened cells were seen (Fig. 1). Some nodules showed central necrosis. Mucicarmine stain showed the presence of intraluminal mucin. There was no evidence of intracellular mucin. PAS stain with diastase showed glycogen in the clear cells in areas. Lobules of minor salivary gland were seen just outside the tumor.. A diagnosis of EMC with predominance of myoepithelial clear cells was suggested. Immunohistochemistry was suggested to rule other clear cell tumors of the salivary gland.

Immunohistochemically, cytokeratin (CK) highlighted the luminal epithelial cells. The myoepithelial cells showed only focal CK positivity. S-100 protein intensely stained the myoepithelial cell cytoplasm and nuclei in about $50 \%$ of the tumour (Fig. 2). Thus, biphasic nature of the tumor confirmed the diagnosis of EMC.

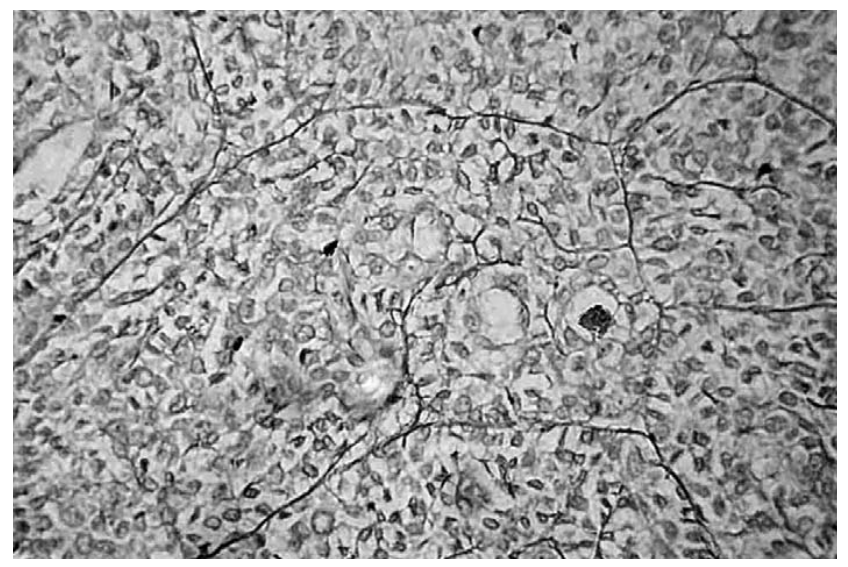

Fig. 1 Solid organoid pattern of clear cells with a few luminal spaces containing mucinous material and lined by flattened cells (Hematoxylin \& Eosin $\times 400$ )
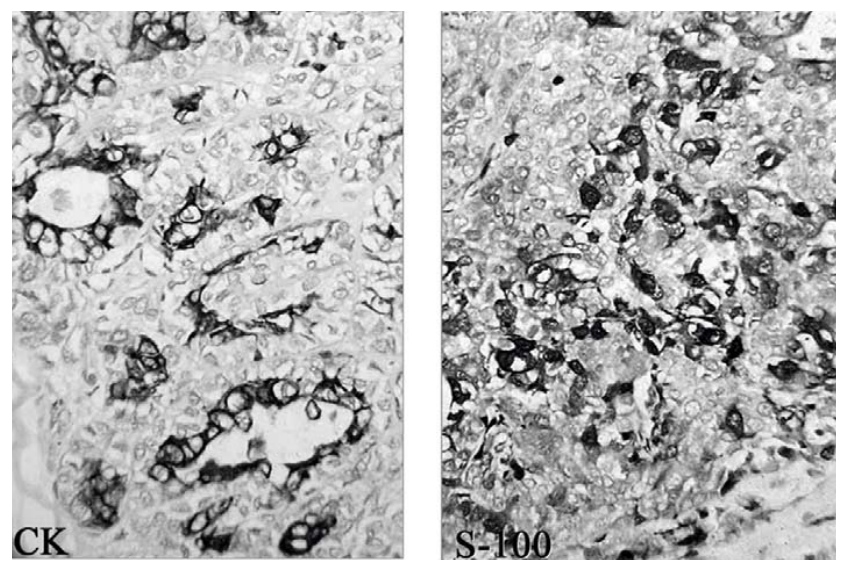

Fig. 2 Immunohistochemistry highlighting the epithelial cells with strong CK positivity. S-100 protein positivity is seen in the myoepithelial cells.

\section{Discussion}

EMC is a multinodular tumor, the nodules separated by dense fibrous connective tissue. Classically, the nodules are made up of well-defined tubules lined by inner cuboidal epithelial cells with pink cytoplasm and outer large clear myoepithelial cells with variable amount of glycogen. The relative proportion of these cells varies from case to case as well as within the same lesion. The architectural variations include solid sheets of myoepithelial cells, organoid nodules, and cystic spaces with papillary projections [1]. When the clear cells predominate with a solid growth pattern, tumors like hyalinising clear cell carcinoma, clear cell variants of mucoepidermoid carcinoma, acinic cell carcinoma and oncocytoma, and, metastatic renal cell carcinoma enter the differential diagnosis $[2,3]$. The characteristic histopathological features in some areas of these tumors enables a differential diagnosis. In the present case, a diagnosis MEC was suggested on the biopsy. However, intracytoplasmic mucin was absent in the excised tumor. IHC is of value to identify the two cell types when they are morphologically indistinct. The above mentioned tumors lack a myoepithelial cell component $[2,3]$. The inconspicuous glandular lumina of EMC can be highlighted by pancytokeratin, epithelial membrane antigen (EMA) and carcinoembryonic antigen (CEA). The myoepithelial cells can show variable and inconsistent $\mathrm{CK}$ immunoreactivity but are EMA and CEA negative [3]. S-100 protein when applied to salivary tissue and salivary neoplasms strongly stains the myoepithelial cell cytoplasm and nuclei [4]. The ductal cells also variably stain for S-100 protein [3]. Smooth muscle actin and myosin, calponin, and, caldesmon are more specific markers for cells of myoepithelial lineage and the ductal cells are negative. The two markers that are ideally indicated for confirmation of EMC are EMA or CEA for epithelial cells and a smooth muscle specific marker for myoepithelial cells.

EMC usually exhibits a low degree of cellular anaplasia and mitotic activity. Its malignant nature is indicated by the infiltrative and destructive growth pattern, foci of necrosis, perineural invasion, frequent recurrences and remote metastases [2]. Some of the features that denote an aggressive course are solid growth pattern, nuclear atypia, DNA aneuploidy, and high proliferative activity [5]. In a study all tumours larger than $3 \mathrm{~cm}$ in their maximum diameter recurred when compared to none that were $<3 \mathrm{~cm}$ in size [6].

Therapeutic approach to these tumors presents a dilemma as there are no definite treatment guidelines. Surgery has been used for treatment of EMC at all sites followed by radiotherapy in some cases. In a patient who refused surgery for EMC of base of the tongue, chemotherapy followed by radiotherapy has shown good results [7] This tumour, though considered as a low grade malignancy, requires regular follow-up due to its tendency to recur and metastasize to distant sites. 


\section{References}

1. Chetty R (2000) Intercalated duct hyperplasia: possible relationship to epithelial-myoepithelial carcinoma and hybrid tumours of salivary gland. Histopathology 37 : 260-263

2. Corio RL, Sciubba JJ, Brannon RB, Batsakis JG (1982) Epithelial-myoepithelial carcinoma of intercalated duct origin. A clinicopathologic and ultrastructural assessment of sixteen cases. Oral Surg Oral Med Oral Pathol $53: 280-287$

3. Rosai J (2004) (ed). Major and Minor Salivary Glands. In: Rosai and Ackerman's Surgical Pathology. 9th edn. India: Elsevier Inc. 873-916

4. Luna MA, Ordonez NG, Mackay B, Batsakis JG, and Guillamondegui O (1985) Salivary epithelial- myoepithelial carcinomas of intercalated ducts: a clinical, electronnmicroscopic, and immunocytochemical study. Oral Surg Oral Med Oral Pathol 59: 482-490

5. Tralongo V, Daniele E (1998) Epithelial-myoepithelial carcinoma of the salivary gland: a review of literature. Anticancer Res 18:603-608

6. Hamper K, Brügmann M, Koppermann R, Caselitz $\mathrm{J}$ et al (1989) Epithelial-myoepithelial duct carcinoma of salivary glands: a follow-up and cytophotometric study of 21 cases. J Oral Pathol Med 18: 299-304

7. Tarun P, Kishore S, Nand SD, Nita K (2004) Epithelial-myoepithelial carcinoma of the base of tongue: Pathology and management. Indian $\mathrm{J}$ Cancer 41:138-140 